\title{
BOUNDARY VALUE PROBLEMS FOR POTENTIALS OF A SINGLE LAYER*
}

BY

E. R. C. MILES

1. Introduction. The principal object of the following paper is the discussion of a Neumann problem, with reference to a potential of a single layer which is based on a general distribution of matter on a simple closed plane boundary. Such potentials were introduced by Plemelj. $\dagger$ The result obtained here is of the same order of generality for these boundaries as that obtained by G. C. Evans with the aid of conformal transformations $\neq$, but the present method is entirely different, and simpler. The problem is equivalent to a Stieltjes integral equation, which is solved by reduction to the classical Fredholm type.

Consider the function

$$
v(M)=\int_{C} \log (1 / r) d \mu(P),
$$

where $\mu(P)$ is a function of limited variation on the simple closed curve $C$. The notation $\mu(P)$ is used as synonymous with $\mu\left(s_{P}\right)$. For the measurement of angles the length $r=M P$ is regarded as directed from $M$ to $P$, where $M$ is a point which remains fixed in the integration. It is assumed that the direction of $C$ changes continuously as we go along the curve, and that the curvature exists at each point.

Let $n$, or, more particularly, $n_{Q}$, be the normal at a point $Q$ of the curve, directed toward the interior, and $M$ a point on $n_{Q}$. The derivative

$$
\frac{d v(M)}{d n}=\int_{C} \frac{\cos \left(n_{Q}, r\right)}{r}-d \mu(P)
$$

generally fails to be continuous as $M$ passes over the point $Q$. We shall investigate this discontinuity.

The integral (2) converges when $M$ is coincident with $Q$. In order to prove this statement, it suffices to examine the integrand in the neighborhood

\footnotetext{
* Presented to the Society, April 7, 1928; received by the editors June 2, 1928.

$\dagger$ Potentialtheoretische Untersuchungen, Leipzig, 1911, p. 17. p. 84 .

$\ddagger$ The Logarithmic Potential. Discontinuous Dirichlet and Neumann Problems, New York, 1927,
} 
of $Q$. Set up a system of rectangular coördinates, with origin at $Q$, and axes along the positive tangent and inner normal, respectively. Measure $s$ from $Q$. In the neighborhood of $Q$, the equation of $C$ may be written in the form

$$
y=x^{2} f(x),
$$

where, as may be verified from the hypotheses for $C, f(x)$ and $x f^{\prime}(x)$ are continuous except at $x=0$, and bounded in a suitable interval containing $Q$.

Denote by $\theta$ the angle measured in the positive direction from the normal at $Q$ to $Q P$. We have then

$$
\frac{\cos \theta}{r}=\frac{y}{r^{2}}=\frac{f(x)}{1+x^{2}[f(x)]^{2}},
$$

which is therefore continuous except at $Q$ as a function of $x$, and bounded in the neighborhood of $Q$. Hence it has the same properties as a function of $s_{P}$ when $P \neq Q$. The statement is therefore proved.

From what has been said of the function $(\cos \theta) / r$, we obtain immediately the following corollary, which will be useful later. We state the result as a lemma.

Lemma. If $\mu(P)$ is continuous at $Q$, and $C^{\prime}$ is an arc of $C$, containing $Q$, and of length $<\delta$, then

$$
\int_{C} \frac{\cos \theta}{r} d \mu(P)=\lim _{\delta \rightarrow 0} \int_{C-C^{\prime}} \frac{\cos \theta}{r} d \mu(P) .
$$

2. Boundary values of the normal derivative. For convenience we denote by $d v(Q+) / d n$ the value, where it exists, of $d v / d n$ as $M$ approaches $Q$ along $n$ from the inside; we define similarly $d v(Q-) / d n$ with reference to the exterior of $C$. With this notation, we have the following theorem:

THEOREM 1. If $v(M)$ is given by (1), then

$$
\begin{aligned}
& \frac{d v(Q+)}{d n}+\frac{d v(Q-)}{d n}=2 \int_{c} \frac{\cos \left(n_{Q}, Q P\right)}{Q P}-d \mu(P), \\
& \frac{d v(Q+)}{d n}-\frac{d v(Q-)}{d n}=-2 \pi \mu^{\prime}(Q),
\end{aligned}
$$

provided $\mu^{\prime}(Q)$ exists.

Denote by $C^{\prime}$ a small $\operatorname{arc} H K$ containing $Q$ as an interior point. We may write (2) in the form

$$
\frac{d v(M)}{d n}=\int_{C-C^{\prime}}+\int_{C^{\prime}} \frac{\cos \theta}{M P} d \mu(P)=I_{1}+I_{2} .
$$


Consider $I_{2}$. Construct the osculating circle $\bar{C}$ at $Q$. Let $\rho$ be the distance from its center to $M, \bar{P}$ that point of $\bar{C}$ for which $x=x_{P}$. Let $\bar{r}=M \bar{P}$, and $\bar{\theta}$ the angle from $n_{Q}$ to $M \bar{P}$, measured in the positive direction. We shall show that

$$
\left|\int_{C^{\prime}}\left[\frac{\cos \theta}{r}-\frac{\cos \bar{\theta}}{\bar{r}}\right] d \mu(P)\right|<\epsilon,
$$

uniformly with respect to $M$, provided that the length of $C^{\prime}$ is small enough. For this purpose it suffices to show that $(\cos \theta) / r-(\cos \bar{\theta}) / \bar{r}$ is bounded uniformly with respect to $M$. In fact, $\mu(P)$ is of limited variation in the neighborhood of $Q$, and continuous at $Q$. The conclusion is apparent if we write

$$
\frac{\cos \theta}{r}-\frac{\cos \bar{\theta}}{\bar{r}}=\frac{(\bar{y}-y) \cos (\theta+\bar{\theta})}{r \bar{r}}
$$

and note (a), that $\bar{y}-y$ is of order higher than the second with respect to $x$, and (b), that $|x|<r, \bar{r}$. The details are as follows:

$$
\bar{y}=R-\left(R^{2}-x^{2}\right)^{1 / 2},
$$

where $R$ is the radius of curvature of $C$ at $Q$. But the preceding equation may also be written

$$
\begin{aligned}
\bar{y} & =R\left(1-\left(1-\frac{x^{2}}{R^{2}}\right)^{1 / 2}\right) \\
& =\frac{x^{2}}{2 R}+x^{4} \Phi(x) .
\end{aligned}
$$

Then

$$
y-\bar{y}=x^{2}\left[f(x)-\frac{1}{2 R}\right]+x^{4} \Phi(x),
$$

where the expression in brackets is bounded. Moreover $\cos (\theta+\bar{\theta})$ is obviously continuous as a function of $x$, and uniformly bounded with respect to $M$. The statement is therefore proved.

Consider therefore the integral

$$
\begin{aligned}
I_{2} & =\int_{\bar{C}^{\prime}} \frac{\cos \bar{\theta}}{\bar{r}} d \mu(P)=\frac{d}{d n} \int_{\bar{C}^{\prime}} \log \frac{1}{\bar{r}} d \mu(P) \\
& =\frac{1}{2} \frac{\partial}{\partial \rho} \int_{\bar{C}^{\prime}} \log \left(R^{2}+\rho^{2}-2 R \rho \cos \psi\right) d \mu(P) \\
& =-\frac{1}{2 \rho} \int_{\bar{C}^{\prime}} \frac{-2 \rho^{2}+2 R \rho \cos \psi}{R^{2}+\rho^{2}-2 R \rho \cos \psi} d \mu(P),
\end{aligned}
$$


where $\psi$ is the angle subtended by $Q \bar{P}$ at the center $O$ of the circle, and where $\rho$ is the segment $O M$, directed from $O$ to $M$. Hence

$$
-I_{2}+\int_{\bar{C}^{\prime}} \frac{1}{2 \rho} d \mu(P)=\frac{1}{2 \rho} \int_{\bar{C}^{\prime}} \frac{R^{2}-\rho^{2}}{R^{2}+\rho^{2}-2 R \rho \cos \psi} d \mu(P) .
$$

If the length of $\bar{C}^{\prime}$ is sufficiently small, we have, therefore,

But*

$$
\left|I_{2}+\frac{1}{2 \rho} \int_{\bar{C}^{\prime}} \frac{R^{2}-\rho^{2}}{R^{2}+\rho^{2}-2 R \rho \cos \psi} d \mu(P)\right|<\epsilon .
$$

$$
\lim _{\rho \rightarrow R} \frac{1}{2 \pi R} \int_{\bar{C}^{\prime}} \frac{R^{2}-\rho^{2}}{R^{2}+\rho^{2}-2 R \rho \cos \psi} d \mu(P)=\mu^{\prime}(Q) .
$$

Hence, taking account of the lemma of $\S 1$,

$$
\frac{d v(Q+)}{d n}=-\pi \mu^{\prime}(Q)+\int_{c} \frac{\cos \left(n_{Q}, Q P\right)}{Q P} d \mu(P),
$$

and similarly

$$
\frac{d v(Q-)}{d n}=\pi \mu^{\prime}(Q)+\int_{c} \frac{\cos \left(n_{Q}, Q P\right)}{Q P} d \mu(P) .
$$

From these equations we have the result stated in the theorem.

The foregoing analysis presupposes that the curvature at $Q$ is not zero. In the latter case, we may replace the arc of the osculating circle by the corresponding segment $\bar{C}^{\prime}$ of the tangent at $Q$, and observe that the integrals along $C^{\prime}$ and along $\bar{C}^{\prime}$ differ by a quantity which is uniformly small with the length of $C^{\prime}$. In fact, $(\cos \theta) / r-(\cos \bar{\theta}) / \bar{r}$ is uniformly bounded with respect to $M$, since $y$ is of order higher than the second with respect to $x$. Therefore consider the integral $\dagger$

$$
\begin{aligned}
& \int_{-x_{0}}^{x_{0}} \frac{\cos \bar{\theta}}{\bar{r}} d \mu(x) \\
& \quad=\mu^{\prime}(Q) \int_{-x_{0}}^{x_{0}} \frac{\cos \bar{\theta}}{\bar{r}} d x-\int_{-x_{0}}^{0} \frac{\bar{y}}{\bar{r}^{2}} d(x \eta(x))-\int_{0}^{x_{0}} \frac{\bar{y}}{\bar{r}^{2}} d(x \eta(x)),
\end{aligned}
$$

where $\mu(x)=x \mu^{\prime}(Q)+x \eta(x)$, the last term being a function of $x$ of limited variation, such that if $\eta_{0}$ denotes the upper bound of $|\eta(x)|$ in the interval $-x_{0} \leqq x \leqq x_{0}$, we have $\lim _{x_{0} \rightarrow 0} \eta_{0}=0$.

* Evans, loc. cit., pp. 39, 40.

$\dagger$ This integral is essentially the Poisson Stieltjes integral for the straight line considered as an infinite circle. 
Now since $\bar{y} / \bar{r}^{2}$ decreases with $x, x>0$, we have

where

$$
\int_{0}^{x_{0}} \frac{\bar{y}}{\bar{r}^{2}} d(x \eta(x))=\frac{\bar{y}}{\bar{r}^{2}} x_{0} \eta\left(x_{0}\right)-\int_{0}^{x_{0}} x \eta(x) d\left(\frac{\bar{y}}{\bar{r}^{2}}\right),
$$

and

$$
\left|\frac{\bar{y}}{\bar{r}^{2}} x_{0} \eta\left(x_{0}\right)\right|<\eta_{0}
$$

$$
\begin{aligned}
\left|\int_{0}^{x_{0}} x \eta(x) d\left(\frac{\bar{y}}{\bar{r}^{2}}\right)\right| & \leqq-\eta_{0} \int_{0}^{x_{0}} x d\left(\frac{\bar{y}}{\bar{r}^{2}}\right) \\
& \leqq \eta_{0} x_{0}\left[\frac{\bar{y}}{\bar{r}^{2}}\right]_{x=x_{0}}+\eta_{0} \int_{0}^{x_{0}} \frac{\bar{y}}{\bar{r}^{2}} d x \\
& \leqq \eta_{0}\left(1+\frac{\pi}{2}\right) .
\end{aligned}
$$

Hence the integral considered is made arbitrarily small with $x_{0}$, uniformly for $M$. The same remark applies to

$$
\int_{-x_{0}}^{0} \frac{\bar{y}}{\bar{r}^{2}} d(x \eta(x))
$$

But $\int_{-x_{0}}^{x_{0}}(\cos \bar{\theta}) / \bar{r} d x=2\left(\theta_{0}-\pi\right)$. Hence, by first taking $x$ small enough, and then taking $M$ near enough to $Q$, we can make

$$
\int_{-x_{0}}^{x_{0}} \frac{\cos \bar{\theta}}{\bar{r}} d \mu(x)
$$

differ by as little as we please from $\pi \mu^{\prime}(Q)$, which is what we wished to show.

3. The Stieltjes integral equation. We consider again the class of functions which can be expressed in the form (1). It is assumed now that the discontinuities of $\mu(P)$ are regular. If we also denote by $C$ the total length of the curve, we may extend the definition of $\mu\left(s_{P}\right)$ beyond $s_{P}=C$ by means of the relation

$$
\mu\left(s_{P}+C\right)=\mu\left(s_{P}\right)+m,
$$

where

$$
m=\int_{C} d \mu\left(s_{P}\right)=\mu(C) .
$$

The problem now to be considered is expressed in terms of the condition

$$
\frac{1+\lambda}{2 \lambda} \lim _{\delta \rightarrow 0+} \int_{A_{\delta}}^{B_{\delta}} \frac{d v}{d n} d s_{\delta}-\frac{1-\lambda}{2 \lambda} \lim _{\delta \rightarrow 0-} \int_{A_{\delta}}^{B_{\delta}} \frac{d v}{d n} d s_{\delta}=g(B)-g(A),
$$


where $g(s)$ is a given function of limited variation, with regular discontinuities, and where $A, B$ are points of $C$. We shall regard $A$ as fixed and $B$ as variable on $C$. In the first integral $s_{b}$ is an arbitrary simple regular curve lying entirely within $C$, and joining the points $A_{\delta}, B_{\delta}$. The latter points are at the distance $\delta$ from $A, B$, measured along the respective inner normals. In the second integral $s_{8}$ lies entirely outside $C$; the points $A_{\delta}, B_{8}$ are on the outer normals at $A, B$, respectively.

That the curve $C$ has a unique normal at each point is implied in the preceding statements. Specifically, it is assumed that $C$ is a simple regular closed curve; moreover $C$ shall be a curve of class $\Gamma,{ }^{*}$ i.e., there shall exist a positive number $\Gamma$, independent of $M$, such that

uniformly for all $M$.

$$
\int_{c} \frac{\left|\cos \left(n_{P}, M P\right)\right|}{M P} d s_{P}<\Gamma,
$$

THEOREM 2. There is a unique function of class (1) which satisfies the condition (3) unless $\lambda$ belongs to a certain set of characteristic values. These values are independent of the given function $g(B)$, and depend merely on the form of the curve $C$.

The value $\lambda=-1$ is not a characteristic value. The corresponding problem is a generalized Neumann problem for the exterior region:

$$
\lim _{\delta \rightarrow 0^{-}} \int_{A_{\delta}}^{B_{\delta}} \frac{d v}{d n} d s_{\delta}=g(B)-g(A) .
$$

The value $\lambda=+1$ is a characteristic value. A necessary and sufficient condition that there exist a solution $v(M)$ of class (1) is that

$$
\int_{C} d g(B)=g(C)=0 .
$$

The corresponding problem is a generalized Neumann problem for the interior region:

$$
\lim _{\delta \rightarrow 0+} \int_{A_{\delta}}^{B_{\delta}} \frac{d v}{d n} d s_{\delta}=g(B)-g(A) .
$$

The solution is unique except for an arbitrary additive constant.

Consider the condition (3). We have

$$
\lim _{\delta \rightarrow 0+} \int_{A_{\delta}}^{B_{\delta}} \frac{d v}{d n} d s_{\delta}=-\lim _{\delta \rightarrow 0+} \int_{A_{\delta}}^{B_{\delta}} \frac{d w}{d s_{\delta}} d s_{\delta}=\lim _{\delta \rightarrow 0+}\left[w\left(A_{\delta}\right)-w\left(B_{\delta}\right)\right],
$$

* G. C. Evans, The Rice Institute Pamphlet, vol. 7 (1920), p. 261. 
where $w$ is a function conjugate to $v$ :

$$
w(M)=-\int_{C} \tan ^{-1} \frac{y_{P}-y_{M}}{x_{P}-x_{M}} d \mu(P) .
$$

The function under the integral sign being multiple valued, we write

$$
w(M)=-\int_{C} \theta(M, P) d \mu(P),
$$

where $\theta(M, P)$ is the angle from $M A$ to $M P$ measured in the positive direction. Then, taking $A$ as the initial point of the integration, $\theta$ varies continuously as $P$ traces the curve.

Integrate by parts:

$$
\begin{aligned}
w(M)= & -[\mu(P) \theta(M, P)]_{A}^{A} \\
& +\int_{C} \mu(P) \frac{d \theta(M, P)}{d s_{P}} d s_{P} \\
= & \phi(M)+u(M) .
\end{aligned}
$$

We shall take $\mu(A)=0$, since this can be effected by the addition of a constant. Then

$$
\phi(M)=\left\{\begin{array}{r}
-2 \pi m, M \text { inside } C \\
0, M \text { outside } C .
\end{array}\right.
$$

The function $u(M)$ is a double layer potential with density a function of limited variation. The integral converges when $M$ is a point on $C$, since $\mu(P)$ is bounded, and $C$ is of class $\Gamma$.

Consider $u(Q+)$ as $M$ approaches a point $Q$ of $C$ along the normal:

$$
u(M)=\int_{C} \mu(P) \frac{d \theta(M, P)}{d s_{P}} d s_{P}=\int_{C} \mu(P) \frac{\cos \left(n_{P}, M P\right)}{M P} d s_{P} .
$$

In order to study the behavior of $u(M)$ in the neighborhood of $Q$, we need to extend the traditional analysis to cover the case where $\mu(P)$ has a regular discontinuity at $Q$.

Write

$$
u(M)=\int_{C-C^{\prime}}+\int_{H}^{Q}+\int_{Q}^{K} \mu(P) \frac{\cos \left(n_{P}, M P\right)}{M P} d s_{P},
$$

where $C^{\prime}$ denotes a small arc $H K$, of which $Q$ is an interior point.

The first of the preceding integrals is continuous at $Q$, since $Q$ is not on $C^{\prime}$. Then

$$
\lim _{M \rightarrow Q} \int_{C-C^{\prime}} \mu(P) \frac{\cos \left(n_{P}, M P\right)}{M P} d s_{P}=\int_{C-C^{\prime}} \mu(P) \frac{\cos \left(n_{P}, Q P\right)}{Q P} d s_{P} .
$$


The second integral may be written

$$
\int_{H}^{Q}[\mu(P)-\mu(Q-)] \frac{\cos \left(n_{P}, M P\right)}{M P} d s_{P}+\mu(Q-) \int_{H}^{Q} \frac{\cos \left(n_{P}, M P\right)}{M P} d s_{P}=I_{1}+I_{2},
$$

where $\lim _{P \rightarrow Q} \mu(P)=\mu(Q-)$, the limit existing since $\mu(P)$ is of limited variation. Hence $H$ may be taken near enough to $Q$ so that $|\mu(P)-\mu(Q-)|<\epsilon / \Gamma$, where $\epsilon>0$ is arbitrarily small. By hypothesis, $C$ is of class $\Gamma$. Consequently

$$
\left|I_{1}\right|<\epsilon
$$

if $s_{H Q}$ is sufficiently small, and uniformly with regard to $M$.

Write $I_{2}$ in the form

$$
\begin{aligned}
I_{2} & =\mu(Q-) \int_{H}^{Q} \frac{d}{d s} \tan ^{-1} \frac{y_{M}-y_{P}}{x_{M}-x_{P}} d s_{P} \\
& =\mu(Q-)\left[\tan ^{-1} \frac{y_{M}-y_{Q}}{x_{M}-x_{Q}}-\tan ^{-1} \frac{y_{M}-y_{H}}{x_{M}-x_{H}}\right] .
\end{aligned}
$$

The quantity within brackets is the angle from $M H$ to $M Q$, measured in the positive direction. As $M$ approaches $Q$ along the normal, this angle approaches as its limit the angle from the chord $Q H$ to the (outer) normal at $Q$. Hence if $H$ is taken near enough to $Q$, the limiting angle may be made as near $\pi / 2$ as we wish. That is,

$$
\lim _{M \rightarrow Q+} I_{2}(M)=\frac{\pi}{2} \mu(Q-)+\eta(H)
$$

where $\lim _{H \rightarrow Q} \eta(H)=0$.

By a similar process we obtain

$$
\begin{aligned}
& \int_{Q}^{K} \mu(P) \frac{\cos \left(n_{P}, M P\right)}{M P} d s_{P} \\
& =\int_{Q}^{K}[\mu(P)-\mu(Q+)] \frac{\cos \left(n_{P}, M P\right)}{M P} d s_{P}+\mu(Q+) \int_{Q}^{K} \frac{\cos \left(n_{P}, M P\right)}{M P} d s_{P} \\
& =I_{3}+I_{4} .
\end{aligned}
$$

By taking $K$ sufficiently near to $Q,\left|I_{3}\right|$ can be made arbitrarily small, uniformly with respect to $M$. Also

$$
\lim _{M \rightarrow Q+} I_{4}(M)=\frac{\pi}{2} \mu(Q+)+\eta^{\prime}(K), \quad \lim _{K \rightarrow Q} \eta^{\prime}(K)=0 .
$$


If $M$ approaches $Q$ along the outer normal at $Q$, the corresponding limits are given by

$$
\begin{aligned}
& \lim _{x \rightarrow Q^{-}} I_{2}^{\prime}(M)=-\frac{\pi}{2} \mu(Q-)+\eta^{\prime \prime}(B), \\
& \lim _{x \rightarrow Q^{-}} I_{4^{\prime}}^{\prime}(M)=-\frac{\pi}{2} \mu(Q+)+\eta^{\prime \prime \prime}(K), \\
& \lim _{B \rightarrow \eta^{\prime \prime}} \eta^{\prime \prime}(B)=\lim _{X \rightarrow Q^{\prime \prime}} \eta^{\prime \prime \prime}(K)=0 .
\end{aligned}
$$

We have, therefore,

$$
\begin{aligned}
u(Q+) & =\int_{c} \mu(P) \frac{\cos \left(n_{P}, Q P\right)}{Q P} d s_{P}+\frac{\pi}{2}[\mu(Q+)+\mu(Q-)] \\
& =\int_{c} \mu(P) \frac{d}{d s_{P}} \tan ^{-1} \frac{y_{Q}-y_{P}}{x_{Q}-x_{P}} d s_{P}+\pi \mu(Q),
\end{aligned}
$$

since the discontinuities of $\mu(P)$ are regular. Similarly

$$
u(Q-)=\int_{C} \mu(P) \frac{d}{d s_{P}} \tan ^{-1} \frac{y_{Q}-y_{P}}{x_{Q}-x_{P}} d s_{P}-\pi \mu(Q) .
$$

If now we take $M$ as $B_{\S}$, we obtain from (4)

$$
\begin{aligned}
& w(B+)+w(B-)=-2 \pi m+2 \int_{c} \mu(P) \frac{d}{d s_{P}} \tan ^{-1} \frac{y_{B}-y_{P}}{x_{B}-x_{P}} d s_{P}, \\
& w(B+)-w(B-)=-2 \pi m+2 \pi \mu(B) .
\end{aligned}
$$

Taking $M$ as $A_{\delta}$,

$$
\begin{aligned}
& w(A+)+w(A-)=-2 \pi m+2 \int_{C} \mu(P) \frac{d}{d s_{P}} \tan ^{-1} \frac{y_{A}-y_{P}}{x_{A}-x_{P}} d s_{P}, \\
& w(A+)-w(A-)=-2 \pi m .
\end{aligned}
$$

We have, therefore,

$$
\begin{aligned}
\lim _{\delta \rightarrow 0+} \int_{\Delta_{\delta}}^{B_{\delta}} \frac{d v}{d n} d s_{\delta} & =w(A+)-w(B+) \\
& =-\pi \mu(B)-\int_{c} \mu(P) \frac{d}{d s_{P}} H(A, B ; P) d s_{P},
\end{aligned}
$$

where

Also

$$
H(A, B ; P)=\tan ^{-1} \frac{y_{B}-y_{P}}{x_{B}-x_{P}}-\tan ^{-1} \frac{y_{A}-y_{P}}{x_{A}-x_{P}} .
$$




$$
\begin{aligned}
\lim _{\delta \rightarrow 0-} \int_{A_{\delta}}^{B_{\delta}} \frac{d v}{d n} d s_{\delta} & =w(A-)-w(B-) \\
& =\pi \mu(B)-\int_{C} \mu(P) \frac{d}{d s_{P}} H(A, B ; P) d s_{P} .
\end{aligned}
$$

Equation (3) may now be written in the form

$$
\mu(B)=-\frac{\lambda}{\pi}[g(B)-g(A)]-\frac{\lambda}{\pi} \int_{C} \mu(P) \frac{d}{d s_{P}}-H(A, B ; P) d s_{P} .
$$

Integrate by parts:

$$
\begin{aligned}
\mu(B)=-\frac{\lambda}{\pi}[g(B)-g(A)]-\frac{\lambda}{\pi}[\mu(P) H(A, B ; P)]_{C} \\
+\frac{\lambda}{\pi} \int_{C} H(A, B ; P) d \mu(P) \\
=-\frac{\lambda}{\pi}[g(B)-g(A)]-m \lambda+\frac{\lambda}{\pi} \int_{C} H(A, B ; P) d \mu(P) .
\end{aligned}
$$

Hence we have the following equation:

$$
\mu(B)=G(B)+\frac{\lambda}{\pi} \int_{C} H(A, B ; P) d \mu(P),
$$

where $G(B)=-(\lambda / \pi)[g(B)-g(A)]-m \lambda$.

If $v(M)$ is a function of class (1) which satisfies (3), the function $\mu(B)$, of limited variation and with regular discontinuities, satisfies the Stieltjes integral equation (5). Conversely, if $\mu(B)$ is a solution of (5), of limited variation and with regular discontinuities, the function $v(M)$ given by (1) will satisfy (3).

4. The equivalent classical equation. We now proceed to show that this equation may be solved by means of one of classical form. Write

$$
\mu(B)=G(B)+R(B) .
$$

We shall prove that if $\mu(B)$ is of limited variation, then $R(B)$ is absolutely continuous.

Substitute the value of $\mu(B)$ from (6) into (5):

(7) $G(B)+R(B)=G(B)+\frac{\lambda}{\pi} \int_{C} H(A, B ; P) d G(P)+\frac{\lambda}{\pi} \int_{C} H(A, B ; P) d R(P)$. 
It suffices to consider one of the above integrals, say the first. We have

$$
\begin{aligned}
H(A, B ; P) & =\int_{A}^{B} \frac{d}{d s_{Q}} \tan ^{-1} \frac{y_{Q}-y_{P}}{x_{Q}-x_{P}} d s_{Q} \\
& =\int_{A}^{B} \frac{\cos \left(n_{Q}, Q P\right)}{Q P} d s_{Q} \\
\int_{C} B(A, B ; P) d G(P) & =\int_{C} d G(P) \int_{A}^{B} \frac{\cos \left(n_{Q}, Q P\right)}{Q P} d s_{Q} .
\end{aligned}
$$

We shall prove that we may invert the order of integration in the right hand member.*

Let $\left\{h_{k}(Q, P)\right\}$ denote a sequence of continuous functions such that

We have

$$
\begin{gathered}
\left.\mid h_{k} Q, P\right) \mid<\frac{\left|\cos \left(n_{Q}, Q P\right)\right|}{Q P} \quad(k=1,2, \cdots), \\
\lim _{k \rightarrow \infty} h_{k}(Q, P)=\frac{\cos \left(n_{Q}, Q P\right)}{Q P} .
\end{gathered}
$$

$$
\int_{C} d G(P) \int_{A}^{B} h_{k}(Q, P) d s_{Q}=\int_{A}^{B} d s_{Q} \int_{C} h_{k}(Q, P) d G(P) .
$$

We shall show first that as $k \rightarrow \infty$ the right hand member of this equation approaches the limit

$$
\int_{A}^{B} d s_{Q} \int_{C} \frac{\cos \left(n_{Q}, Q P\right)}{Q P} d G(P) .
$$

For this purpose we define a sequence $\left\{q_{m}(Q, P)\right\}$ as follows:

$$
\begin{aligned}
q_{m}(Q, P) & =\frac{\left|\cos \left(n_{Q}, Q P\right)\right|}{Q P}, \text { if } \frac{\left|\cos \left(n_{Q}, Q P\right)\right|}{Q P}<m, \\
& =m, \quad \text { otherwise. }
\end{aligned}
$$

The sequence

$$
\int_{C} q_{m}(Q, P) d T(P)
$$

* The proof is essentially that in Evans, The Rice Institute Pamphlet, vol. 7 (1920), p. 263, but is given here in more detail, for the sake of clearness. 
where $T$ is the total variation function of $G(P)$, is non-decreasing with $m$. Also

$$
\int_{A}^{B} d s_{Q} \int_{C} q_{m}(Q, P) d T(P)=\int_{C} d T(P) \int_{A}^{B} q_{m}(Q, P) d s_{Q}<\Gamma T(C)
$$

and is therefore bounded for all $m$. Consequently

$$
\lim _{m \rightarrow \infty} \int_{c} q_{m}(Q, P) d T(P)=\int_{c} \frac{\left|\cos \left(n_{Q}, Q P\right)\right|}{Q P} d T(P)
$$

exists nearly everywhere on $C$, and is a summable function. Moreover, from $\left(8^{\prime}\right)$,

$$
\int_{A}^{B} d s_{Q} \int_{C} \frac{\left|\cos \left(n_{Q}, Q P\right)\right|}{Q P} d T(P)=\int_{C} d T(P) \int_{A}^{B} \frac{\left|\cos \left(n_{Q}, Q P\right)\right|}{Q P} d s_{Q},
$$

by the fundamental properties of the Lebesgue and Daniell integrals.

Consequently, from the definition of $h_{k}(Q, P)$, we have

$$
\lim _{k \rightarrow \infty} \int_{C} h_{k}(Q, P) d G(P)=\int_{C} \frac{\cos \left(n_{Q}, Q P\right)}{Q P} d G(P),
$$

the integral on the right being a Daniell integral, which by $\left(8^{\prime}\right)$ represents a summable function. Moreover

$$
\left|\int_{C} h_{k}(Q, P) d G(P)\right| \leqq \int_{C} \frac{\left|\cos \left(n_{Q}, Q P\right)\right|}{Q P} d T(P),
$$

a summable function. Hence, by the Lebesgue limit theorem,

$$
\lim _{k \rightarrow \infty} \int_{A}^{B} d s_{Q} \int_{C} h_{k}(Q, P) d G(P)=\int_{A}^{B} d s_{Q} \int_{C} \frac{\cos \left(n_{Q}, Q P\right)}{Q P} d G(P) .
$$

As for the left hand side of $(8),{ }^{*}$ we have

$$
\lim _{k \rightarrow \infty} \int_{A}^{B} h_{k}(Q, P) d s_{Q}=\int_{A}^{B} \frac{\cos \left(n_{Q}, Q P\right)}{Q P} d s_{Q} .
$$

In fact $\int_{C} d T(P) \int_{A}^{B} h_{k}(Q, P) d s_{Q}$ exists for all $k$. Moreover $\int_{C} d T(P)$ $\cdot \int_{A}^{B}\left[\left|\cos \left(n_{Q}, Q P\right)\right| /(Q P)\right] d s_{Q}$ exists, since, as we have seen, the inside integral is a summable function. Finally

$$
\int_{A}^{B}\left|h_{k}(Q, P)\right| d s_{Q}<\int_{A}^{B} \frac{\left|\cos \left(n_{Q}, Q P\right)\right|}{Q P} d s_{Q} .
$$

* Evans, The Rice Institute Pamphlet, vol. 7 (1920), p. 257. 
Consequently $\int_{C} d G(P) \int_{A}^{B}\left[\cos \left(n_{Q}, Q P\right) /(Q P)\right] d s_{Q}$ exists and

$$
\lim _{k \rightarrow \infty} \int_{C} d G(P) \int_{A}^{B} h_{k}(Q, P) d s_{Q}=\int_{C} d G(P) \int_{A}^{B} \frac{\cos \left(n_{Q}, Q P\right)}{Q P} d s_{Q} .
$$

We obtain therefore from (8), as $k \rightarrow \infty$,

$$
\int_{C} d G(P) \int_{A}^{B} \frac{\cos \left(n_{Q}, Q P\right)}{Q P} d s_{Q}=\int_{A}^{B} d s_{Q} \int_{C} \frac{\cos \left(n_{Q}, Q P\right)}{Q P} d G(P) .
$$

The right hand side, being the indefinite integral of a summable function, is absolutely continuous. So therefore is the left hand side. Similarly for the remaining integral in (7). Hence $R(B)$ is absolutely continuous. If then we write (7) in the form

$$
R(B)=\frac{\lambda}{\pi} \int_{C} H(A, B ; P)[d G(P)+d R(P)],
$$

the derivative nearly everywhere of $R(B)$ is

$$
r(B)=\frac{\lambda}{\pi} \int_{C} \frac{\cos \left(n_{B}, B P\right)}{B P}-[d G(P)+d R(P)] .
$$

Let $h(B)=(\lambda / \pi) \int_{C}\left[\cos \left(n_{B}, B P\right) /(B P)\right] d G(P)$. Then, since $R(B)$ is absolutely continuous:

we have

$$
R(B)=\int_{A}^{B} r(Q) d s_{Q}
$$

$$
r(B)=h(B)+\frac{\lambda}{\pi} \int_{c} \frac{\cos \left(n_{B}, B P\right)}{B P} r(P) d s_{P} .
$$

Thus (9) is a consequence of (5). Conversely, if (9) has a summable solution $r(B)$, then $\mu(B)$ given by (6) is of limited variation and a solution of (5).

But (9) has a unique solution unless $\lambda$ is one of a set of characteristic values, since (9) is the classical integral equation for the Neumann problem. Moreover it is known that $\lambda=-1$ is not a characteristic value for this equation.

We know that $\lambda=+1$ is a characteristic value, and that a necessary and sufficient condition that (9) have a solution is that

$$
\int_{c} h(Q) d s_{Q}=0
$$

But 


$$
\int_{C} h(Q) d s_{Q}=\frac{1}{\pi} \int_{C} d s_{B} \int_{C} \frac{\cos \left(n_{B}, B P\right)}{B P} d G(P) .
$$

By changing the order of integration,

$$
\frac{1}{\pi} \int_{C} d G(P) \int_{C} \frac{\cos \left(n_{B}, B P\right)}{B P} d s_{B}=\frac{1}{\pi} \int_{C} \pi d G(P)=G(C)=0 .
$$

Hence $g(C)=0$. Conversely, if $G(C)=0$, the analysis may be retraced, and we have (10). The condition $g(C)=0$ is therefore necessary and sufficient in order that (5) have a solution for $\lambda=+1$.

The function $v(M)$ determined by (1) is therefore that belonging to a particular solution of (5) by means of (1) plus the function determined by (1) where the $\mu(P)$ is a solution of the homogeneous equation corresponding to (5). But in this case

$$
\mu(Q)=\int_{A}^{Q} \psi(P) d s_{P}
$$

where $\psi(P)$ is a solution of the homogeneous equation corresponding to (9). But we know the functions $v(M)=\int_{C} \log (1 /(M P)) \psi(P) d s_{P}$ to be constant. Theorem 2 is therefore proved.

In $\$ \S 3,4$ we have assumed only that $C$ is of class $\Gamma$, and have not required the existence of a curvature as in $\$ \$ 1,2 .^{*}$

The foregoing methods may be applied to obtain analogous theorems for potentials of a double layer, not only for the plane, but also for the threedimensional case. The latter case, both for the single and the double layer, is to be discussed in a paper which the author is writing in conjunction with Professor Evans, who suggested the present investigation.

\footnotetext{
* If the curve has a curvature which is bounded for all points of the curve, then it is automatically of class $\Gamma$.

The Rice Institute, Houston, Texas
} 